\title{
Síndrome de Gilbert
}

\author{
Morales, E.*; Llorens, R.** \\ * Médico de la Unidad de Conductas Adictivas de Catarroja. \\ * Médico de la Unidad de Conductas Adictivas de Alzira.
}

Enviar correspondencia:

Esperanza Morales. C/ Paco Pierra, 1-52ª .46017 VALENCIA. e-mail: emorales@ctv.es

\section{Sr. Director:}

Deseamos participar a los lectores la experiencia de un paciente joven, adicto a heroína fumada, con abusos de alcohol y cocaína en fin de semana, que en la primera evaluación clínica presentaba ictericia y cuya inclusión en tratamiento hizo revertir el cuadro ictérico.

El paciente, de veintiséis años de edad, convive con su familia nuclear, ocupa un puesto de trabajo semicualificado aceptablemente remunerado, y consulta por hábito adictivo. En la anamnesis reconoce un consumo diario de 1/4 a 1/2 g. de heroína fumada, de al menos 17 meses continuados de evolución. Tres intentos anteriores por abandonar este consumo resultaron infructuosos, dos de ellos mediante tratamiento con naltrexona. Además, algunos fines de semana esnifa $1 / 2 \mathrm{~g}$. de cocaína y bebe entre 7 y 8 UBE's de alcohol (70 - 80 g.). Y fuma cada día 1 - 2 "canutos". El perfil psicológico durante la evaluación impresionó de normalidad y la familia confirmó que su conducta y adaptación eran aceptablemente satisfactorias, a excepción de conflictos puntuales ocasionados por los consumos.

La exploración clínica reveló un tinte ictérico con absoluta normalidad del resto. Y refiere que desde su relación con las drogas se ha notado "los ojos amarillos".

Nuestros exámenes complementarios ofrecieron una función hepática normal y objetivaron la elevación de bilirrubina total ( $<$ de $4 \mathrm{mg} / \mathrm{dl}$ ), a expensas de la indirecta (87\%). Todo ello en ausencia clínica de hemólisis.

Incluimos al paciente en un tratamiento de mantenimiento de metadona (TMM) con una dosis de 35 $\mathrm{mg} / \mathrm{día}$ y psicoterapia individual y de pareja. Y le explicamos la necesidad de abstinencia al alcohol, junto con el beneficio de una dieta equilibrada y con el aporte calórico adecuado a sus necesidades.
Tras dos meses de tratamiento las orinas permanecen negativas a opiáceos y cocaína, el consumo de alcohol se ha reducido a "alguna cerveza sin" en fin de semana, pero mantiene el de THC. Las cifras de bilirrubina se normalizaron rápidamente y permanecen sin cambios. Y, según explica, ha mejorado su relación familiar.

Ante un paciente con ictericia y una bilirrubina total no superior a $5 \mathrm{mg} / \mathrm{dl}$., casi toda ella indirecta $(80 \%)$, que coincide con: estrés quirúrgico o ejercicio intenso, o consumo de alcohol, o fiebre, o infecciones, o ayuno prolongado, en ausencia de síntomas generales y que conserva una función hepática normal, debe pensarse en el síndrome de Gilbert (1).

El síndrome de Gilbert se debe a un déficit parcial del enzima glucoroniltransferasa acompañado o no de hemólisis oculta (1). Recientes estudios de genética molecular han confirmado que el fenotipo clínico del síndrome de Gilbert puede describirse como un polimorfismo dinucleótido, que afecta a la función promotora del gen TATA, para el enzima bilirrubin-uridindifosfo- glucoronosiltransferasa (UGT-1A1) (2). La expresión fenotípica del Gilbert es mucho menos frecuente que el genotipo. Y para que éste se exprese, a menudo, son necesarios factores ambientales que ponen en evidencia el déficit genético. El alcohol exige al hígado un esfuerzo adicional para su metabolización, por esta razón puede actuar precipitando la hiperbilirrubinemia en pacientes con Gilbert genotípico, no fenotíticamente expresado (3).

La importancia de este hallazgo también reside en comprender que, la deficiencia en la glucoronoconjugación hepática, afecta al metabolismo de numerosos fármacos que, aún a dosis habituales, podrían ver amplificados sus efectos adversos (3). 


\section{BIBLIOGRAFÍA}

(1) Isselbacher KJ. Metabolismo de la bilirrubina e hiperbilirrubinemia. En: Isselbacher kJ, Braunwald E, Wilson JD, Martin JB, Fauci AS, Kasper DL, editores. Harrison: Principios de Medicina Interna (13 ${ }^{\mathrm{a}}$ edición) Madrid McGraw-Hill - Interamericana de España. 1994:
$1670-1676$.

(2) Burchell B, Hume R. Molecular genetic basis of Gilbet's syndrome. J Gastroenterol Hepatol 1999; 14 (10): 960 $-966$.

(3) Tukey RH, Strassburg CP. Human UDP-glucoronosyltransferases: metabolism, expression, and disease. Annu Rev Pharmacol Toxicol 2000; 40: 581 - 616. 


\title{
¿Pregunta sobre violencia a sus pacientes alcohólicos?
}

\author{
Martín Centeno, A.*; Rojano Capilla, P.** \\ * Médico de familia. \\ * Psiquiatra. Centro de Salud Mental Miraflores. Alcobendas. Área V de Madrid.
}

Enviar correspondencia:

Antonio Martín Centeno. Plaza de la Encina, 2, 3º-3. Alcobendas. 28100 Madrid. Teléfono 91 663.87.52.

\section{Sr. Director:}

Los efectos que el consumo de alcohol produce sobre el juicio de realidad y sobre la personalidad convierten al sujeto en fuente de conflictos con el medio que pueden manifestarse como actos violentos. Cuando el alcohólico se desinhibe con el consumo, pueden aparecer conductas agresivas que se dirigen principalmente hacia sus familiares $(1,2)$. En un estudio reciente encontraron que hasta en las dos terceras partes de los delitos violentos está implicado el alcohol(3).

Existe una gran alarma social en lo que respecta a los maltratos en la vida doméstica, en los que en muchos casos se ven entremezcladas las conductas violentas y el consumo de alcohol $(2,4,5)$.

Nuestro objetivo era determinar la frecuencia de conductas violentas en pacientes alcohólicos derivados desde Atención Primaria, para valorar la utilidad de realizar preguntas específicamente sobre violencia a la hora de realizar la historia clínica a un paciente alcohólico en la consulta.

Realizamos un estudio descriptivo transversal en pacientes derivados a un Centro de Salud Mental desde Atención Primaria con objeto de medir en estos la frecuencia de conductas violentas, perfil sociodemográfico e historia de alcoholismo. La muestra fue de 40 pacientes y fue tomada de pacientes nuevos diagnosticados de problemas relacionados con alcohol en un periodo de seis meses, realizándose la entrevista por el médico en la primera consulta. El diagnóstico de alcoholismo se realizó según criterios del DSM-IV. En dicha entrevista se realizó un cuestionario sociodemográfico y una encuesta para detectar conductas violentas, con preguntas sacadas de los test de impulsividad y violencia de Plutckick validados por Gabriel Rubio.
Algunas de las preguntas sobre violencia realizadas fueron:

$-¿$ Tiene usted comportamientos agresivos o violentos?

-Si los tiene: ¿los tiene en su casa o fuera?

-Cuando está agresivo: ¿llega a ser violencia física, o sólo verbal?

- ¿Alguna vez ha lastimado o pegado a un familiar?

$-¿ Y$ a alguien que no sea familiar suyo?

- ¿Le han detenido alguna vez?

- ¿Podría conseguir un arma con facilidad?

La media de edad fue de 38 años, siendo varones el $85 \%$. Todos eran dependientes del alcohol con un consumo medio de 10 unidades de bebida estándar (UBE) al día (1 UBE=10 gramos de alcohol puro).

Las conductas violentas eran reconocidas en el $91 \%$ de los pacientes. Desglosando en violencia verbal y física, la segunda ocurría en el $40 \%$. Al preguntar por violencia en el hogar esta era reconocida en el $75 \%$. El 17\% reconocían haber pegado en alguna ocasión a un familiar.

El porcentaje de sujetos que habían tenido problemas legales fue de un 23\%, habiendo sido detenidos el $20 \%$. La mitad de los sujetos afirmaba que podría conseguir un arma con facilidad.

Hasta un $10 \%$ de la población española puede tener problemas con su consumo de alcohol. Este consumo se asocia con conductas violentas en muchos casos(2). En nuestra muestra hemos encontrado datos que nos hacen pensar que en la mayoría de los pacientes alcohólicos existen conductas violentas, centradas en ocasiones en el ámbito doméstico. Por ello es importante realizar preguntas encaminadas a detectar específicamente estas conductas en 
los pacientes alcohólicos y en sus familias. Creemos que es importante realizar entrevistas con las familias e intervenir en ellas cuando estas conductas existan, derivando a través de nuestra asistente social a equipos especializados. En el tratamiento de un alcohólico(6) es fundamental el apoyo familiar, sin él, las expectativas de éxito son escasas o nulas. Los maltratos y las conductas violentas constituyen un elemento desestructurador de la familia. El abordaje ha de ser multidisciplinar y requiere coordinación entre los equipos especializados en el apoyo a las familias y los que están en la actualidad tratando a los pacientes alcohólicos(7).

\section{BIBLIOGRAFÍA}

1. Rojano Capilla, Ferre Navarrete. Aspectos legales relacionados con el alcohol. En: G. Rubio Valladolid. Guía práctica de intervención en alcoholismo. Agencia Antidroga de la Comunidad de Madrid. Madrid. 2000.

2. Kyriacou et al. Risk factor for injury from domestic violence against women. N Engl J Med 1999.16;341:1892-8.

3. Pérez Martín J. Delitos de violencia relacionados con el alcohol. III Jornadas de la Sociedad Española de Psiquiatría Legal. Editorial Nuevo Siglo, S.L. Noviembre 1995.

4. Miller BA, Smyth NJ, Mudar PJ. Mother's alcohol and others drugs problems and their punitiveness toward their children. J Stud Alcohol 1999;60:632-42.

5. Leonard KE, Quigley BM. Drinking and marital aggression in newlyweds: an event based analysis of drinking and the occurrence of husband marital aggression. J Stud Alcohol 1999;60:537-45.

6. Morales Rueda A, Rubio Valladolid. Diagnóstico y tratamiento de los problemas relacionados con el alcohol en Atención Primaria. MEDIFAM 1997;7:211-225.

7.Problemas por el alcohol en la familia. Informe de la Unión Europea. Madrid: Comisión Europea. Dirección General V. Agencia Antidroga de la Comunidad de Madrid. 1999. 